\title{
On superstability of exponential functional equations
}

\author{
Batool Noori ${ }^{1}$, M.B. Moghimi ${ }^{1 *}$, Abbas Najati ${ }^{1}$, Choonkil Park2 ${ }^{2^{*}}$ (D) and Jung Rye Lee ${ }^{3 *}$ (D)
}

\section{"Correspondence:}

mbfmoghimi@yahoo.com;

baak@hanyang.ac.kr;

jrlee@daejin.ac.kr

'Department of Mathematics, Faculty of Sciences, University of Mohaghegh Ardabili, Ardabil, Iran ${ }^{2}$ Research Institute for Natural

Sciences, Hanyang University, Seoul 04763, Korea

${ }^{3}$ Department of Data Science, Daejin University, Kyunggi 11159, Korea

\author{
Abstract \\ The aim of this paper is to prove the superstability of the following functional \\ equations:

$$
f(P(x, y))=g(x) h(y)
$$$$
f(x+y)=g(x) h(y) .
$$ \\ MSC: 39B72; 39B82; 39B52 \\ Keywords: Banach algebra; Pexider exponential equation; Exponential function; \\ Superstability
}

\section{Introduction and preliminaries}

The stability problem of functional equations was raised by Ulam from a question concerning the stability of group homomorphisms [1]. Hyers [2] obtained the first important result in this field. See [3-10] for more information on functional equations and applications. In 1979, Baker, Lawrence, and Zorzitto [11] proved the superstability of the exponential functional equation: Let $X$ be a real vector space and $f: X \rightarrow \mathbb{R}$ be an approximately exponential function, i.e., there exists a nonnegative number $\varepsilon$ such that

$$
\|f(x+y)-f(x) f(y)\| \leq \varepsilon, \quad x, y \in X .
$$

Then $f$ is either bounded or exponential. The same result is also true for approximately exponential mappings $f$ from a semigroup $(G,+)$ with values in a normed algebra with the property that the norm is multiplicative [12]. Gavvruta [13] proved the superstability of the Lobacevski functional equation

$$
f\left(\frac{x+y}{2}\right)^{2}=f(x) f(y)
$$

(c) The Author(s) 2021. This article is licensed under a Creative Commons Attribution 4.0 International License, which permits use, sharing, adaptation, distribution and reproduction in any medium or format, as long as you give appropriate credit to the original author(s) and the source, provide a link to the Creative Commons licence, and indicate if changes were made. The images or other third party material in this article are included in the article's Creative Commons licence, unless indicated otherwise in a credit line to the material. If material is not included in the article's Creative Commons licence and your intended use is not permitted by statutory regulation or exceeds the permitted use, you will need to obtain permission directly from the copyright holder. To view a copy of this licence, visit http://creativecommons.org/licenses/by/4.0/. 
under the condition bounded by a constant. Kim [14] investigated the solution and the superstability of the Pexiderized Lobacevski functional equation

$$
f\left(\frac{x+y}{2}\right)^{2}=g(x) h(y)
$$

Kim and Park [15] considered the superstability of the generalized Pexider exponential functional equation

$$
f\left(\frac{x+y}{m}\right)^{m}=g(x) h(y)
$$

in unital normed algebras, where $m$ is a positive integer. For more information on the superstability of functional equations and applications, see [16-18].

The aim of this paper is to prove the superstability of the following generalized Pexider exponential functional equation:

$$
f(P(x, y))=g(x) h(y)
$$

in unital normed algebras.

\section{Superstability of the generalized Pexider exponential functional equation (GPE)}

In this section, assume that $(G, *)$ is a semigroup with identity $e, A$ is a commutative unital normed algebra with unit $I$, and $P: G \times G \rightarrow G$ is a function such that

$$
P(x, y * z)=P(x * y, z), \quad x, y, z \in G .
$$

It is clear that if $x * y=y * x$, then $P(x, y)=P(x, y * e)=P(x * y, e)=P(y, x)$.

\section{Example 2.1}

(1) Let $G$ be an operator algebra and $P: G \times G \rightarrow G$ be given by $P(a, b)=a b$ for all $a, b \in G$. Then $P(x, y z)=x(y z)=(x y) z=P(x y, z)$ for all $x, y, z \in G$.

(2) Let $G=G L_{2}(\mathbb{C})$ be the set of invertible $2 \times 2$ complex matrices and $P: G \times G \rightarrow G$ be given by $P(a, b)=b^{-1} a^{-1}$ for all $a, b \in G$. Then

$$
P(x, y z)=(y z)^{-1} x^{-1}=\left(z^{-1} y^{-1}\right) x^{-1}=z^{-1}\left(y^{-1} x^{-1}\right)=z^{-1}(x y)^{-1}=P(x y, z)
$$

for all $x, y, z \in G$.

(3) Let $G=U(A)$ be the unitary group of a unital $C^{*}$-algebra $A$ and $P: G \times G \rightarrow G$ be given by $P(a, b)=b^{*} a^{*}$ for all $a, b \in G$. Then

$$
P(x, y z)=(y z)^{*} x^{*}=\left(z^{*} y^{*}\right) x^{*}=z^{*}\left(y^{*} x^{*}\right)=z^{*}(x y)^{*}=P(x y, z)
$$

for all $x, y, z \in G$ (see [19]).

We prove the superstability of the generalized Pexider exponential equation (GPE). 
Theorem 2.2 Let $\varphi: G \times G \rightarrow[0,+\infty)$ be a function. Assume that $\sup _{y \in G} \varphi(x, y)<\infty$ for each $x \in G$, and that $f, g, h: G \rightarrow A$ satisfy the inequality

$$
\|f(P(x, y))-g(x) h(y)\| \leq \varphi(x, y)
$$

for all $x, y \in G$. If there exists a sequence $\left\{y_{n}\right\}_{n}$ in $G$ such that $\left\|h\left(y_{n}\right)^{-1}\right\| \rightarrow 0$ as $n \rightarrow \infty$, then $g$ satisfies $g(x * y) g(e)=g(x) g(y)$ for all $x, y \in G$.

Proof It follows from (2.1) that

$$
\begin{aligned}
\left\|f\left(P\left(x, y_{n}\right)\right) h\left(y_{n}\right)^{-1}-g(x)\right\| & =\left\|\left[f\left(P\left(x, y_{n}\right)\right)-g(x) h\left(y_{n}\right)\right] h\left(y_{n}\right)^{-1}\right\| \\
& \leq\left\|f\left(P\left(x, y_{n}\right)\right)-g(x) h\left(y_{n}\right)\right\| \cdot\left\|h\left(y_{n}\right)^{-1}\right\| \\
& \leq\left\|h\left(y_{n}\right)^{-1}\right\| \varphi\left(x, y_{n}\right)
\end{aligned}
$$

for all $x \in G$. So we have

$$
g(x)=\lim _{n \rightarrow \infty} f\left(P\left(x, y_{n}\right) h\left(y_{n}\right)^{-1}, \quad x \in G .\right.
$$

For $x, y \in G$, let $\Delta(x, y)=f(P(x, y))-g(x) h(y)$. Then (2.1) implies that

$$
\left\|\Delta\left(x, y * y_{n}\right) g(z)\right\| \leq \varphi\left(x, y * y_{n}\right)\|g(z)\|
$$

for all $x, y, z \in G$. Since $\lim _{n \rightarrow \infty}\left\|h\left(y_{n}\right)^{-1}\right\|=0$, we get

$$
\lim _{n \rightarrow \infty} \Delta\left(x, y * y_{n}\right) g(z) h\left(y_{n}\right)^{-1}=0, \quad x, y, z \in G .
$$

Therefore it follows from (2.5) that

$$
\begin{aligned}
g(x * y) g(z)= & \lim _{n \rightarrow \infty} f\left(P\left(x * y, y_{n}\right) h\left(y_{n}\right)^{-1} g(z)\right. \\
= & \lim _{n \rightarrow \infty} f\left(P\left(x, y * y_{n}\right) h\left(y_{n}\right)^{-1} g(z)\right. \\
= & \lim _{n \rightarrow \infty}\left[\Delta\left(x, y * y_{n}\right) h\left(y_{n}\right)^{-1} g(z)+g(x) h\left(y * y_{n}\right) h\left(y_{n}\right)^{-1} g(z)\right] \\
= & \lim _{n \rightarrow \infty}\left[\Delta\left(x, y * y_{n}\right) g(z) h\left(y_{n}\right)^{-1}+f\left(P\left(z, y * y_{n}\right)\right) g(x) h\left(y_{n}\right)^{-1}\right. \\
& \left.-\Delta\left(z, y * y_{n}\right) g(x) h\left(y_{n}\right)^{-1}\right] \\
= & \lim _{n \rightarrow \infty}\left[\Delta\left(x, y * y_{n}\right) g(z) h\left(y_{n}\right)^{-1}+f\left(P\left(z * y, y_{n}\right)\right) g(x) h\left(y_{n}\right)^{-1}\right. \\
& \left.-\Delta\left(z, y * y_{n}\right) g(x) h\left(y_{n}\right)^{-1}\right] \\
= & g(x) g(z * y) .
\end{aligned}
$$

Hence

$$
g(x * y) g(z)=g(x) g(z * y), \quad x, y, z \in G .
$$

Putting $z=e$ in (2.4), we obtain $g(x * y) g(e)=g(x) g(y)$ for all $x, y \in G$. 
Using the proof of Theorem 2.2, we get the following result.

Theorem 2.3 Let $\varphi: G \times G \rightarrow[0,+\infty)$ be a function. Assume that $f, g, h: G \rightarrow A$ satisfy inequality (2.1) for all $x, y \in G$. If there exists a sequence $\left\{y_{n}\right\}_{n}$ in $G$ such that $\varphi(x, y *$ $\left.y_{n}\right)\left\|h\left(y_{n}\right)^{-1}\right\| \rightarrow 0$ for all $x, y \in G$, as $n \rightarrow \infty$, then $g$ satisfies $g(x * y) g(e)=g(x) g(y)$ for all $x, y \in G$.

Proof According to the proof of Theorem 2.2, we get (2.2). By the assumption (with $y=e$ ), we have $\varphi\left(x, y_{n}\right)\left\|h\left(y_{n}\right)^{-1}\right\| \rightarrow 0$ for all $x \in G$. Then (2.2) implies (2.5). Let $\Delta(x, y):=$ $f(P(x, y))-g(x) h(y)$. By $(2.1)$, we have

$$
\left\|\Delta\left(x, y * y_{n}\right) g(z)\right\| \leq \varphi\left(x, y * y_{n}\right)\|g(z)\|, \quad x, y, z \in G .
$$

Since $\varphi\left(x, y * y_{n}\right)\left\|h\left(y_{n}\right)^{-1}\right\| \rightarrow 0$, we get

$$
\lim _{n \rightarrow \infty} \Delta\left(x, y * y_{n}\right) g(z) h\left(y_{n}\right)^{-1}=0, \quad x, y, z \in G
$$

The rest of the proof is the same as the proof of Theorem 2.2.

The proof of the following theorem is similar to the proof of Theorem 2.2.

Theorem 2.4 Let $\varphi: G \times G \rightarrow[0,+\infty)$ be a function. Assume that $\sup _{x \in G} \varphi(x, y)<\infty$ for each $y \in G$, and that $f, g, h: G \rightarrow A$ satisfy inequality (2.1) for all $x, y \in G$. If there exists a sequence $\left\{y_{n}\right\}_{n}$ in $G$ such that $\left\|g\left(y_{n}\right)^{-1}\right\| \rightarrow 0$ as $n \rightarrow \infty$, then $h$ satisfies $h(x * y) h(e)=$ $h(x) h(y)$ for all $x, y \in G$.

Proof It follows from (2.1) that

$$
\begin{aligned}
\| f & \left(P\left(y_{n}, x\right)\right) g\left(y_{n}\right)^{-1}-h(x) \| \\
& =\left\|\left[f\left(P\left(y_{n}, x\right)\right)-g\left(y_{n}\right) h(x)\right] g\left(y_{n}\right)^{-1}\right\| \\
& \leq\left\|f\left(P\left(y_{n}, x\right)\right)-g\left(y_{n}\right) h(x)\right\| \cdot\left\|g\left(y_{n}\right)^{-1}\right\| \\
& \leq\left\|g\left(y_{n}\right)^{-1}\right\| \varphi\left(y_{n}, x\right)
\end{aligned}
$$

for all $x \in G$. So we have

$$
h(x)=\lim _{n \rightarrow \infty} f\left(P\left(y_{n}, x\right) g\left(y_{n}\right)^{-1}, \quad x \in G .\right.
$$

Let $\Delta(x, y)=f(P(x, y))-g(x) h(y)$. Then (2.1) implies that

$$
\left\|\Delta\left(y_{n} * y, z\right) h(x)\right\| \leq \varphi\left(y_{n} * y, z\right)\|h(x)\|, \quad x, y, z \in G .
$$

Since $\lim _{n \rightarrow \infty}\left\|g\left(y_{n}\right)^{-1}\right\|=0$, we get

$$
\lim _{n \rightarrow \infty} \Delta\left(y_{n} * y, z\right) h(x) g\left(y_{n}\right)^{-1}=0, \quad x, y, z \in G
$$


Therefore it follows from (2.5) that

$$
\begin{aligned}
h(x * y) h(z) & =\lim _{n \rightarrow \infty} f\left(P\left(y_{n}, x * y\right) g\left(y_{n}\right)^{-1} h(z)\right. \\
& =\lim _{n \rightarrow \infty} f\left(P\left(y_{n} * x, y\right) g\left(y_{n}\right)^{-1} h(z)\right. \\
& =\lim _{n \rightarrow \infty}\left[\Delta\left(y_{n} * x, y\right) h(z) g\left(y_{n}\right)^{-1}+g\left(y_{n} * x\right) h(y) g\left(y_{n}\right)^{-1} h(z)\right] \\
& =\lim _{n \rightarrow \infty}\left[\Delta\left(y_{n} * x, y\right) h(z) g\left(y_{n}\right)^{-1}+f\left(P\left(y_{n} * x, z\right)\right) h(y) g\left(y_{n}\right)^{-1}\right. \\
& \left.-\Delta\left(y_{n} * x, z\right) h(y) g\left(y_{n}\right)^{-1}\right] \\
& =\lim _{n \rightarrow \infty} f\left(P\left(y_{n}, x * z\right)\right) g\left(y_{n}\right)^{-1} h(y) \\
& =h(x * z) h(y) .
\end{aligned}
$$

Hence

$$
h(x * y) h(z)=h(x * z) h(y), \quad x, y, z \in G .
$$

Letting $z=e$ in (2.6), we obtain $h(x * y) h(e)=h(x) h(y)$ for all $x, y \in G$.

Remark 2.5 It is clear that the results in Theorems 2.2 and 2.4 are valid if $\varphi(x, y)=\varepsilon$ for all $x, y \in G$, where $\varepsilon \geq 0$ is a constant.

Corollary 2.6 Let $\varphi: G \times G \rightarrow[0,+\infty)$ be a function. Assume that $\sup _{y \in G} \varphi(x, y)<\infty$ for each $x \in G\left(\sup _{x \in G} \varphi(x, y)<\infty\right.$ for each $\left.y \in G\right)$, and that $f, g: G \rightarrow$ A satisfy the inequality

$$
\|f(P(x, y))-g(x) g(y)\| \leq \varphi(x, y)
$$

for all $x, y \in G$ and $g(e)=I$. If there exists a sequence $\left\{y_{n}\right\}_{n}$ in $G$ such that $\left\|g\left(y_{n}\right)^{-1}\right\| \rightarrow 0$ as $n \rightarrow \infty$, then $g$ satisfies $g(x * y)=g(x) g(y)$ for all $x, y \in G$.

Proof Letting $h=g$ in Theorems 2.2 and 2.4 and using $g(e)=I$, we get the desired result.

Corollary 2.7 Let $\varphi: G \times G \rightarrow[0,+\infty)$ be a function. Assume that $\sup _{y \in G} \varphi(x, y)<\infty$ for each $x \in G\left(\sup _{x \in G} \varphi(x, y)<\infty\right.$ for each $\left.y \in G\right)$, and that $f: G \rightarrow A$ satisfies the inequality

$$
\|f(P(x, y))-f(x) f(y)\| \leq \varphi(x, y)
$$

for all $x, y \in G$. If there exists a sequence $\left\{y_{n}\right\}_{n}$ in $G$ such that $\left\|f\left(y_{n}\right)^{-1}\right\| \rightarrow 0$ as $n \rightarrow \infty$, then $f$ satisfies $f(x * y) f(e)=f(x) f(y)$ for all $x, y \in G$.

Proof Letting $h=g=f$ in Theorems 2.2 and 2.4, we get the desired result.

Corollary 2.8 Let $\varphi: G \times G \rightarrow[0,+\infty)$ be a function. Assume that $\sup _{y \in G} \varphi(x, y)<\infty$ for each $x \in G$, and that $f, g, h: G \rightarrow \mathbb{C}$ satisfy the inequality

$$
|f(P(x, y))-g(x) h(y)| \leq \varphi(x, y)
$$

for all $x, y \in G$. If $h$ is not bounded, then $g$ satisfies $g(x * y) g(e)=g(x) g(y)$ for all $x, y \in G$. 
Proof Since $h$ is not bounded, one can choose $\left\{y_{n}\right\}_{n}$ such that $\left|h\left(y_{n}\right)^{-1}\right|=\frac{1}{\left|h\left(y_{n}\right)\right|} \rightarrow 0$ as $n \rightarrow \infty$. Hence one can get the desired result by Theorem 2.2.

Corollary 2.9 Let $\varphi: G \times G \rightarrow[0,+\infty)$ be a function. Assume that $\sup _{x \in G} \varphi(x, y)<\infty$ for each $y \in G$, and that $f, g, h: G \rightarrow \mathbb{C}$ satisfy the inequality

$$
|f(P(x, y))-g(x) h(y)| \leq \varphi(x, y)
$$

for all $x, y \in G$. If $g$ is not bounded, then $h$ satisfies $h(x * y) h(e)=h(x) h(y)$ for all $x, y \in G$.

Proof Since $g$ is not bounded, one can choose $\left\{y_{n}\right\}_{n}$ such that $\left|g\left(y_{n}\right)^{-1}\right|=\frac{1}{\left|g\left(y_{n}\right)\right|} \rightarrow 0$ as $n \rightarrow \infty$. Hence one can get the desired result by Theorem 2.4.

Corollary 2.10 Suppose that $f: G \rightarrow \mathbb{C}$ satisfies the inequality

$$
|f(P(x, y))-f(x) f(y)| \leq \varepsilon
$$

for all $x, y \in G$. Iff is not bounded, then $f$ satisfies $f(x * y) f(e)=f(x) f(y)$ for all $x, y \in G$.

Remark 2.11 When the semigroup $G$ and the function $\varphi(x, y)$ in the above results are replaced with an algebra $B$ and $\varphi(x)$ or $\varphi(y)$, respectively, we have similar results.

Given a semigroup $(G, *)$ and a commutative field $\mathbb{F}$, let $W$ be a vector space of functions $f$ from $G$ into $\mathbb{F}$. The vector space $W$ is called right invariant if, for each $f \in W$, the mapping $\psi_{y}: G \rightarrow \mathbb{F}$, for each $y \in G$, defined by $\psi_{y}(x)=f(x * y)$ belongs to $W$. Left invariant spaces are defined similarly. The vector space $W$ is called invariant if it is both right and left invariant. Following Székelyhidi [20], we obtain the following results.

Theorem 2.12 Given a semigroup $(G, *)$ with identity e, a commutative field $\mathbb{F}$, and a right invariant vector space $W$ of $\mathbb{F}$-valued functions on $G$. Let $f, g, h: G \rightarrow \mathbb{F}$ be such that the function $\psi_{y}: G \rightarrow \mathbb{F}$ defined by $\psi_{y}(x)=f(P(x, y))-g(x) h(y)$ belongs to $W$ for each $y \in G$. If $h(e)=1$, then either $g \in W$ or $h(x * y)=h(x) h(y)$ for all $x, y \in G$.

Proof Suppose that there are $y_{0}, z_{0} \in G$ such that $h\left(y_{0} * z_{0}\right) \neq h\left(y_{0}\right) h\left(z_{0}\right)$. Hence

$$
\begin{aligned}
{\left[h\left(y_{0} * z_{0}\right)-h\left(y_{0}\right) h\left(z_{0}\right)\right] g(x)=} & {\left[f\left(P\left(x * y_{0}, z_{0}\right)\right)-g\left(x * y_{0}\right) h\left(z_{0}\right)\right] } \\
& -\left[f\left(P\left(x, y_{0} * z_{0}\right)\right)-g(x) h\left(y_{0} * z_{0}\right)\right] \\
& +h\left(z_{0}\right)\left[f\left(P\left(x, y_{0}\right)\right)-g(x) h\left(y_{0}\right)\right] \\
& -h\left(z_{0}\right)\left[f\left(P\left(x * y_{0}, e\right)\right)-g\left(x * y_{0}\right) h(e)\right]
\end{aligned}
$$

for all $x \in G$. Since $\psi_{y} \in W$ for each $y \in G$, we get that the function $\phi_{y, z}: G \rightarrow \mathbb{F}$ defined by $\phi_{y, z}(x)=\psi_{y}(x * z)$ for all $z \in G$ belongs to $W$. Therefore

$$
g=\left[h\left(y_{0} * z_{0}\right)-h\left(y_{0}\right) h\left(z_{0}\right)\right]^{-1}\left[\phi_{z_{0}, y_{0}}-\phi_{y_{0} * z_{0}, e}+h\left(z_{0}\right) \phi_{y_{0}, e}-h\left(z_{0}\right) \phi_{e, y_{0}}\right] .
$$

So we conclude $g \in W$. 
Let $(G, *)$ be a semigroup and $B(G, \mathbb{C})$ be the linear space of bounded functions with complex values on $G$. It is clear that $B(G, \mathbb{C})$ is an invariant vector space. Hence Corollary 2.9 is a consequence of Theorem 2.12.

Theorem 2.13 Given a semigroup $(G, *)$ with identity e, a commutative field $\mathbb{F}$, and an invariant vector space $W$ of $\mathbb{F}$-valued functions on $G$. Let $f, g, h: G \rightarrow \mathbb{F}$ be such that the functions $\phi_{x}, \psi_{y}: G \rightarrow \mathbb{F}$ defined by $\phi_{x}(y)=f(P(x, y))-g(x) h(y)$ and $\psi_{y}(x)=f(P(x, y))-$ $g(x) h(y)$ belong to $W$ for each $x, y \in G$. If $h(e)=1$, then either $g \in W$ or $h(x * y)=h(x) h(y)$ and $g(x)=g(e) h(x)$ for all $x, y \in G$.

Proof Suppose that $g \notin W$. By Theorem 2.12, $h$ satisfies $h(x * y)=h(x) h(y)$ for all $x, y \in G$. Since $\phi_{e} \in W$ and $W$ is left invariant, the function $\xi_{x}: G \rightarrow \mathbb{F}$ for each $x \in G$, defined by $\xi_{x}(y)=\phi_{e}(x * y)$, belongs to $W$. For all $x, y \in G$, we have

$$
\begin{aligned}
f(P(e, x * y))-g(e) h(x * y) & =[f(P(x, y))-g(x) h(y)]+[g(x) h(y)-g(e) h(x * y)] \\
& =[f(P(x, y))-g(x) h(y)]+[g(x)-g(e) h(x)] h(y) .
\end{aligned}
$$

Therefore

$$
[g(x)-g(e) h(x)] h=\xi_{x}-\phi_{x}, \quad x \in G
$$

We claim that $g(x)=g(e) h(x)$ for all $x \in G$. If there is $x_{0} \in G$ such that $g\left(x_{0}\right)-g(e) h\left(x_{0}\right) \neq$ 0 , then (2.7) implies that $h \in W$. Since $h, \phi_{e} \in W$, we get that the function $\vartheta: G \rightarrow \mathbb{F}$ defined by $\vartheta(y)=f(P(e, y))=f(P(y, e))$ belongs to $W$. Therefore $g \in W$, since $\vartheta=\psi_{e}+g$. This contradiction implies that $g(x)=g(e) h(x)$ for all $x \in G$.

Corollary 2.14 Given a semigroup $(G, *)$ with identity e and a function $\varphi: G \times G \rightarrow$ $[0,+\infty)$ with $\sup _{x \in G} \varphi(x, y)<\infty$ and $\sup _{y \in G} \varphi(x, y)<\infty$ for all $x, y \in G$. Let $f, g, h: G \rightarrow \mathbb{F}$ be such that

$$
|f(P(x, y))-g(x) h(y)| \leq \varphi(x, y), \quad x, y \in G
$$

If $h(e)=1$, then either $g$ is bounded or $h(x * y)=h(x) h(y)$ and $g(x)=g(e) h(x)$ for all $x, y \in G$.

Proof Let $W$ be the vector space of all bounded functions from $G$ to $\mathbb{F}$. Then $W$ is an invariant vector space. Hence the desired result follows from Theorem 2.13.

Corollary 2.15 Given a semigroup $(G, *)$ with identity e, a commutative field $\mathbb{F}$, and an invariant vector space $W$ of $\mathbb{F}$-valued functions on $G$. Let $f, g, h: G \rightarrow \mathbb{F}$ be such that the functions $\phi_{x}, \psi_{y}: G \rightarrow \mathbb{F}$ defined by $\phi_{x}(y)=f(x * y)-g(x) h(y)$ and $\psi_{y}(x)=f(x * y)-g(x) h(y)$ belong to $W$ for each $x, y \in G$. If $h(e)=1$, then either $g \in W$ or $h(x * y)=h(x) h(y)$ and $g(x)=g(e) h(x)$ for all $x, y \in G$. Moreover, if $g \in W$, then $f \in W$.

Proof The desired result follows from Theorem 2.13 by letting $P(x, y)=x * y$ for all $x, y \in G$.

Specially, we have the following result without any assumption on $h$. 
Corollary 2.16 Given a semigroup $(G, *)$ with identity e, a commutative field $\mathbb{F}$, and an invariant vector space $W$ of $\mathbb{F}$-valued functions on $G$. Let $f, h: G \rightarrow \mathbb{F}$ be such that the functions $\phi_{x}, \psi_{y}: G \rightarrow \mathbb{F}$ defined by $\phi_{x}(y)=f(x * y)-f(x) h(y)$ and $\psi_{y}(x)=f(x * y)-f(x) h(y)$ belong to $W$ for each $x, y \in G$. Then either $f \in W$ or $h(x * y)=h(x) h(y)$ and $f(x)=f(e) h(x)$ for all $x, y \in G$.

Proof Suppose that $f \notin W$. We claim that $h(x * y)=h(x) h(y)$ for all $x, y \in G$. Let $y_{0}, z_{0} \in G$ such that $h\left(y_{0} * z_{0}\right) \neq h\left(y_{0}\right) h\left(z_{0}\right)$. Hence

$$
\begin{aligned}
{\left[h\left(y_{0} * z_{0}\right)-h\left(y_{0}\right) h\left(z_{0}\right)\right] f(x)=} & {\left[f\left(x * y_{0} * z_{0}\right)-f\left(x * y_{0}\right) h\left(z_{0}\right)\right] } \\
& -\left[f\left(x * y_{0} * z_{0}\right)-f(x) h\left(y_{0} * z_{0}\right)\right] \\
& +h\left(z_{0}\right)\left[f\left(x * y_{0}\right)-f(x) h\left(y_{0}\right)\right]
\end{aligned}
$$

for all $x \in G$. Since $\psi_{y} \in W$ for each $y \in G$, we get that the function $\theta_{y, z}: G \rightarrow \mathbb{F}$ defined by $\theta_{y, z}(x)=\psi_{y}(x * z)$ for all $z \in G$ belongs to $W$. Therefore

$$
f=\left[h\left(y_{0} * z_{0}\right)-h\left(y_{0}\right) h\left(z_{0}\right)\right]^{-1}\left[\theta_{z_{0}, y_{0}}-\theta_{y_{0} * z_{0}, e}+h\left(z_{0}\right) \theta_{y_{0}, e} .\right.
$$

So we conclude $f \in W$, which is a contradiction. Now, we prove $f(x)=f(e) h(x)$ for all $x \in G$. Suppose that there is $x_{0} \in G$ such that $f\left(x_{0}\right)-f(e) h\left(x_{0}\right) \neq 0$. For each $y \in G$, we have

$$
\begin{aligned}
\left.f\left(x_{0} * y\right)\right)-f(e) h\left(x_{0} * y\right) & =\left[f\left(x_{0} * y\right)-f\left(x_{0}\right) h(y)\right]+\left[f\left(x_{0}\right) h(y)-f(e) h\left(x_{0} * y\right)\right] \\
& =\left[f\left(x_{0} * y\right)-f\left(x_{0}\right) h(y)\right]+\left[f\left(x_{0}\right)-f(e) h\left(x_{0}\right)\right] h(y) .
\end{aligned}
$$

Therefore, $\left[g\left(x_{0}\right)-f(e) h\left(x_{0}\right)\right] h \in W$, and so $h \in W$. Since $h, \phi_{e} \in W$ and $\phi_{e}=f-f(e) h$, we get $f \in W$, which is again a contradiction.

Theorem 2.17 Given a semigroup $(G, *)$, a commutative field $\mathbb{F}$, and a right invariant vector space $W$ of $\mathbb{F}$-valued functions on $G$. Let $f, g, h: G \rightarrow \mathbb{F}$ be such that the function $\psi_{y}: G \rightarrow \mathbb{F}$ defined by $\psi_{y}(x)=f(x * y)-g(x) h(y)$ belongs to $W$ for each $y \in G$. If $f-g \in W$ or $h(e)=1$ (when $G$ has the identity e), then either $f, g \in W$ or $h(x * y)=h(x) h(y)$ for all $x, y \in G$.

Proof Let $G$ have not an identity. Suppose that $f-g \in W$ and there are $y_{0}, z_{0} \in G$ such that $h\left(y_{0} * z_{0}\right) \neq h\left(y_{0}\right) h\left(z_{0}\right)$. Then

$$
\begin{aligned}
{\left[h\left(y_{0} * z_{0}\right)-h\left(y_{0}\right) h\left(z_{0}\right)\right] g(x)=} & {\left[f\left(x * y_{0} * z_{0}\right)-g\left(x * y_{0}\right) h\left(z_{0}\right)\right] } \\
& -\left[f\left(x * y_{0} * z_{0}\right)-g(x) h\left(y_{0} * z_{0}\right)\right] \\
& +h\left(z_{0}\right)\left[f\left(x * y_{0}\right)-g(x) h\left(y_{0}\right)\right] \\
& -h\left(z_{0}\right)\left[f\left(x * y_{0}\right)-g\left(x * y_{0}\right)\right]
\end{aligned}
$$

for all $x \in G$. Since $\psi_{y}, f-g \in W$ for each $y \in G$, we get that the functions $\phi_{y}, \varphi: G \rightarrow \mathbb{F}$ defined by $\phi_{y}(x)=\psi_{y}\left(x * y_{0}\right)$ and $\varphi(x)=(f-g)\left(x * y_{0}\right)$ belong to $W$. Therefore

$$
g=\left[h\left(y_{0} * z_{0}\right)-h\left(y_{0}\right) h\left(z_{0}\right)\right]^{-1}\left[\phi_{z_{0}}-\psi_{y_{0} * z_{0}}+h\left(z_{0}\right) \psi_{y_{0}}-h\left(z_{0}\right) \varphi\right] .
$$


So $g \in W$, and we conclude $f \in W$. For $h(e)=1$, if $G$ has the identity $e$, we get $f-g \in W$ by the hypothesis. Hence the result follows from the previous case.

Specially, we get Székelyhidi’s result.

Corollary 2.18 ([20]) Given a semigroup $(G, *)$, a commutative field $\mathbb{F}$, and a right invariant vector space $W$ of $\mathbb{F}$-valued functions on $G$. Let $f, g: G \rightarrow \mathbb{F}$ be such that the function $\psi_{y}: G \rightarrow \mathbb{F}$ defined by $\psi_{y}(x)=f(x * y)-f(x) g(y)$ belongs to $W$ for each $y \in G$. Then either $f \in W$ or $g(x * y)=g(x) g(y)$ for all $x, y \in G$.

Proof It follows from Theorem 2.17 by replacing $g$ with $f$, and $h$ with $g$.

\section{Superstability of the Pexider exponential equation}

Using an idea from [21], we establish the superstability of the Pexider exponential equation $f(x+y)=g(x) h(y)$.

Theorem 3.1 Let $X$ and $E$ be a real normed space and a normed algebra with multiplicative norm, respectively. Let $a \in(E \backslash\{0\}) \cup(\mathbb{R} \backslash\{0\})$ and $f: X \rightarrow E$ be a function such that $a f(z)=f(z)$ a for all $z \in X$. Iff satisfies the inequality

$$
\|a f(x+y)-f(x) f(y)\| \leq \varepsilon\left(\|x\|^{p}+\|y\|^{p}\right)+\theta\|x\|^{p}\|y\|^{p}, \quad x, y \in X
$$

for some $\varepsilon, \theta, p \geq 0$, then either $\sup _{\|x\| \geq 1} \frac{\|f(x)\|}{\|x\|^{p}}<\infty$ or

$$
a f(x+y)=f(x) f(y), \quad x, y \in X
$$

Proof We assume that $\varepsilon+\theta>0$ and continue to employ the notation $\sharp a \sharp$ to denote $\|a\|$ (if $a \in E$ ) and $|a|$ (if $a \in \mathbb{R}$ ), respectively. Let $\left\{\frac{\|f(x)\|}{\|x\|^{p}}:\|x\| \geq 1\right\}$ be not bounded. Then there exists a sequence $\left\{x_{n}\right\}_{n=1}^{\infty} \subseteq X$ such that

$$
\left\|x_{n}\right\| \geq 1, \quad \frac{\left\|f\left(x_{n}\right)\right\|}{\left\|x_{n}\right\|^{p}} \geq n, \quad n \in \mathbb{N}
$$

Therefore

$$
\lim _{n \rightarrow \infty} \frac{\left\|x_{n}\right\|^{p}}{\left\|f\left(x_{n}\right)\right\|}=0, \quad \lim _{n \rightarrow \infty}\left\|f\left(x_{n}\right)\right\|=+\infty .
$$

Choose $x, y, z \in X$ with $f(x) \neq 0$. It then follows from (3.1) that

$$
\begin{aligned}
\|a f(x+y+z)-f(z) f(x+y)\| & \leq \varepsilon\left(\|x+y\|^{p}+\|z\|^{p}\right)+\theta\|x+y\|^{p}\|z\|^{p}, \\
\|a f(x+y+z)-f(y+z) f(x)\| & \leq \varepsilon\left(\|y+z\|^{p}+\|x\|^{p}\right)+\theta\|y+z\|^{p}\|x\|^{p} .
\end{aligned}
$$

Hence we get

$$
\begin{aligned}
\|f(z) f(x+y)-f(y+z) f(x)\| \leq & \varepsilon\left(\|x+y\|^{p}+\|y+z\|^{p}+\|x\|^{p}+\|z\|^{p}\right) \\
& +\theta\left(\|x+y\|^{p}\|z\|^{p}+\|y+z\|^{p}\|x\|^{p}\right) .
\end{aligned}
$$


In view of (3.1), we have

$$
\|a f(z) f(x+y)-f(z) f(y) f(x)\| \leq\|f(z)\|\left[\varepsilon\left(\|x\|^{p}+\|y\|^{p}\right)+\theta\|x\|^{p}\|y\|^{p}\right]
$$

Inequalities (3.3) and (3.4) yield

$$
\begin{aligned}
\|a f(y+z) f(x)-f(z) f(y) f(x)\| \leq & \varepsilon \sharp a \sharp\left(\|x+y\|^{p}+\|y+z\|^{p}+\|x\|^{p}+\|z\|^{p}\right) \\
& +\theta \sharp a \sharp\left(\|x+y\|^{p}\|z\|^{p}+\|y+z\|^{p}\|x\|^{p}\right) \\
& +\|f(z)\|\left[\varepsilon\left(\|x\|^{p}+\|y\|^{p}\right)+\theta\|x\|^{p}\|y\|^{p}\right] .
\end{aligned}
$$

Since $E$ is a normed algebra with multiplicative norm, it follows from (3.5) that

$$
\begin{aligned}
\|a f(y+z)-f(z) f(y)\| \\
\leq \frac{\varepsilon \sharp a \sharp\left(\|x+y\|^{p}+\|y+z\|^{p}+\|x\|^{p}+\|z\|^{p}\right)}{\|f(x)\|} \\
+\frac{\theta \sharp a \sharp\left(\|x+y\|^{p}\|z\|^{p}+\|y+z\|^{p}\|x\|^{p}\right)}{\|f(x)\|} \\
+\frac{\|f(z)\|\left[\varepsilon\left(\|x\|^{p}+\|y\|^{p}\right)+\theta\|x\|^{p}\|y\|^{p}\right]}{\|f(x)\|} .
\end{aligned}
$$

If we put $x=x_{n}$ in (3.6) and take the limit as $n \rightarrow+\infty$, then it follows from (3.2) that

$$
a f(z+y)=f(z) f(y)
$$

as desired.

Theorem 3.2 Let $X$ and $E$ be a real normed space and a normed algebra with multiplicative norm, respectively. Let $f, g: X \rightarrow E$ be functions such that $f(0) f(z)=f(z) f(0)$ for all $z \in X$ and satisfy

$$
\|g(x+y)-f(x) f(y)\| \leq \varepsilon\left(\|x\|^{p}+\|y\|^{p}\right)+\theta\|x\|^{p}\|y\|^{p}, \quad x, y \in E
$$

for some $\varepsilon, \theta, p \geq 0$. Then either $\sup _{\|x\| \geq 1} \frac{\|g(x)\|}{\|x\|^{p}}<\infty$ or

$$
f(0) f(x+y)=f(x) f(y), \quad x, y \in X
$$

Proof Let $\left\{\frac{\|g(x)\|}{\|x\|^{p}}:\|x\| \geq 1\right\}$ be not bounded. Then (3.7) implies that $f(0) \neq 0$ and $\left\{\frac{\|f(x)\|}{\|x\|^{p}}\right.$ : $\|x\| \geq 1\}$ is not bounded. In view of (3.7), we have

$$
\|g(x+y)-f(0) f(x+y)\| \leq \varepsilon\|x+y\|^{p} \leq 2^{p} \varepsilon\left(\|x\|^{p}+\|y\|^{p}\right), \quad x, y \in X
$$

Therefore

$$
\begin{aligned}
\|f(0) f(x+y)-f(x) f(y)\| & \leq\|f(0) f(x+y)-g(x+y)\|+\|g(x+y)-f(x) f(y)\| \\
& \leq\left(2^{p}+1\right) \varepsilon\left(\|x\|^{p}+\|y\|^{p}\right)+\theta\|x\|^{p}\|y\|^{p}, \quad x, y \in X .
\end{aligned}
$$

By Theorem 3.1, we conclude that $f(0) f(x+y)=f(x) f(y)$ for all $x, y \in X$. 
Theorem 3.3 Let $X$ and $E$ be a real normed space and a normed algebra with multiplicative norm, respectively. Let $a \in(E \backslash\{0\}) \cup(\mathbb{R} \backslash\{0\})$ and $f, g: X \rightarrow E$ be functions satisfying one of the following conditions:

(i) $a f(z)=f(z) a,\|a f(x+y)-f(x) g(y)\| \leq \varepsilon\left(\|x\|^{p}+\|y\|^{p}\right)+\theta\|x\|^{p}\|y\|^{p}, x, y, z \in X$;

(ii) $a g(z)=g(z) a,\|a f(x+y)-g(x) f(y)\| \leq \varepsilon\left(\|x\|^{p}+\|y\|^{p}\right)+\theta\|x\|^{p}\|y\|^{p}, x, y, z \in X$, for some $\varepsilon, \theta, p \geq 0$. Then either $\sup _{\|x\| \geq 1} \frac{\|f(x)\|}{\|x\|^{p}}<\infty$ or

$$
a g(x+y)=g(x) g(y), \quad x, y \in X .
$$

Proof We use the notation $\sharp a \sharp$ to denote $\|a\|$ (if $a \in E$ ) and $|a|$ (if $a \in \mathbb{R}$ ), respectively. Let $f$, $g$ satisfy (i) and $\left\{\frac{\|f(x)\|}{\|x\|^{P}}:\|x\| \geq 1\right\}$ be unbounded. Then there exists a sequence $\left\{x_{n}\right\}_{n=1}^{\infty} \subseteq X$ such that (3.2) holds true. In view of (i), we have

$$
\begin{aligned}
& \|a f(x+y+z)-f(x+y) g(z)\| \leq \varepsilon\left(\|x+y\|^{p}+\|z\|^{p}\right)+\theta\|x+y\|^{p}\|z\|^{p}, \\
& \|a f(x+y+z)-f(x) g(y+z)\| \leq \varepsilon\left(\|x\|^{p}+\|y+z\|^{p}\right)+\theta\|x\|^{p}\|y+z\|^{p}, \quad x, y, z \in X .
\end{aligned}
$$

Therefore

$$
\begin{aligned}
\|f(x+y) g(z)-f(x) g(y+z)\| \leq & \varepsilon\left(\|x+y\|^{p}+\|y+z\|^{p}+\|x\|^{p}+\|z\|^{p}\right) \\
& +\theta\left(\|x+y\|^{p}\|z\|^{p}+\|x\|^{p}\|y+z\|^{p}\right)
\end{aligned}
$$

for all $x, y, z \in X$. On the other hand, (i) implies

$$
\|a f(x+y) g(z)-f(x) g(y) g(z)\| \leq\|g(z)\|\left[\varepsilon\left(\|x\|^{p}+\|y\|^{p}\right)+\theta\|x\|^{p}\|y\|^{p}\right]
$$

for all $x, y, z \in X$. It follows from (3.8) and (3.9) that

$$
\begin{aligned}
\|a f(x) g(y+z)-f(x) g(y) g(z)\| \leq & \varepsilon \sharp a \sharp\left(\|x+y\|^{p}+\|y+z\|^{p}+\|x\|^{p}+\|z\|^{p}\right) \\
& +\theta \sharp a \sharp\left(\|x+y\|^{p}\|z\|^{p}+\|x\|^{p}\|y+z\|^{p}\right) \\
& +\|g(z)\|\left[\varepsilon\left(\|x\|^{p}+\|y\|^{p}\right)+\theta\|x\|^{p}\|y\|^{p}\right]
\end{aligned}
$$

for all $x, y, z \in X$. Since $a f(x)=f(x) a$ and $E$ is a normed algebra with multiplicative norm, it follows from (3.10) that

$$
\begin{aligned}
\|a g(y+z)-g(y) g(z)\| & \leq \frac{\varepsilon \sharp a \sharp\left(\|x+y\|^{p}+\|y+z\|^{p}+\|x\|^{p}+\|z\|^{p}\right)}{\|f(x)\|} \\
& +\frac{\theta \sharp a \sharp\left(\|x+y\|^{p}\|z\|^{p}+\|x\|^{p}\|y+z\|^{p}\right)}{\|f(x)\|} \\
& +\frac{\|g(z)\|\left[\varepsilon\left(\|x\|^{p}+\|y\|^{p}\right)+\theta\|x\|^{p}\|y\|^{p}\right]}{\|f(x)\|}
\end{aligned}
$$

for all $x, y, z \in X$. If we put $x=x_{n}$ in (3.11) and take the limit as $n \rightarrow+\infty$, then it follows from (3.2) that

$$
a g(z+y)=g(z) g(y), \quad y, z \in X
$$

Similarly, we get the result if $f, g$ satisfy condition (ii). 
Theorem 3.4 Let $X$ and $E$ be a real normed space and a normed algebra with multiplicative norm, respectively. Let $f, g, h: X \rightarrow E$ be functions satisfying the inequality

$$
\|f(x+y)-g(x) h(y)\| \leq \varepsilon\left(\|x\|^{p}+\|y\|^{p}\right)+\theta\|x\|^{p}\|y\|^{p}, \quad x, y \in X
$$

for some $\varepsilon, \theta, p \geq 0$.

(i) If $g(0) h(x)=h(x) g(0)$ for all $x \in X$, then either $\sup _{\|x\| \geq 1} \frac{\|h(x)\|}{\|x\|^{p}}<\infty$ or

$$
g(0) g(x+y)=g(x) g(y), \quad x, y \in X
$$

(ii) If $h(0) g(x)=g(x) h(0)$ for all $x \in X$, then either $\sup _{\|x\| \geq 1} \frac{\|g(x)\|}{\|x\|^{p}}<\infty$ or

$$
h(0) h(x+y)=h(x) h(y), \quad x, y \in X .
$$

Proof In view of (3.12), we have

$$
\begin{aligned}
& \|f(x+y)-g(0) h(x+y)\| \leq \varepsilon\|x+y\|^{p} \leq 2^{p} \varepsilon\left(\|x\|^{p}+\|y\|^{p}\right), \\
& \|f(x+y)-g(x+y) h(0)\| \leq \varepsilon\|x+y\|^{p} \leq 2^{p} \varepsilon\left(\|x\|^{p}+\|y\|^{p}\right), \quad x, y, z \in X .
\end{aligned}
$$

Therefore

$$
\begin{aligned}
& \|g(0) h(x+y)-g(x) h(y)\| \\
& \quad \leq\|f(x+y)-g(0) h(x+y)\|+\|f(x+y)-g(x) h(y)\| \\
& \quad \leq\left(2^{p}+1\right) \varepsilon\left(\|x\|^{p}+\|y\|^{p}\right)+\theta\|x\|^{p}\|y\|^{p},
\end{aligned}
$$

and

$$
\begin{aligned}
& \|g(x+y) h(0)-g(x) h(y)\| \\
& \quad \leq\|f(x+y)-g(x+y) h(0)\|+\|f(x+y)-g(x) h(y)\| \\
& \quad \leq\left(2^{p}+1\right) \varepsilon\left(\|x\|^{p}+\|y\|^{p}\right)+\theta\|x\|^{p}\|y\|^{p}
\end{aligned}
$$

for all $x, y, z \in X$. To prove (i), if $g(0) \neq 0$, the result follows by Theorem 3.3. For the case $g(0)=0$, inequality $(3.13)$ yields

$$
\|g(x) h(y)\| \leq\left(2^{p}+1\right) \varepsilon\left(\|x\|^{p}+\|y\|^{p}\right)+\theta\|x\|^{p}\|y\|^{p}, \quad x, y \in X .
$$

Hence, if $\left\{\frac{\|h(x)\|}{\|x\|^{p}}:\|x\| \geq 1\right\}$ is unbounded, then the last inequality implies that $g(x)=0$ for all $x \in X$. This completes the proof of (i).

Similarly, one can prove (ii).

We now show some counterparts of Shtern's theorem (see [7]).

Theorem 3.5 Let E be a normed linear space and $\mathcal{A}$ be a complex Banach algebra. Assume that $a \in \mathcal{A} \cup \mathbb{R}$ and the mapping $f: E \rightarrow \mathcal{A}$ is such that af $(z)=f(z)$ a for all $z \in E$, and

$$
\|a f(x+y)-f(x) f(y)\| \leq \varepsilon\left(\|x\|^{p}+\|y\|^{p}\right)+\theta\|x\|^{p}\|y\|^{p}, \quad x, y \in E
$$


for some $\varepsilon, \theta, p \geq 0$. If, for each nonzero element $b \in \mathcal{A}$, the E-orbit of $b$

$$
O_{R E}(f, b):=\left\{\frac{f(x) b}{\|x\|^{p}}: x \in E,\|x\| \geq 1\right\}, \quad \text { or } \quad O_{L E}(b, f):=\left\{\frac{b f(x)}{\|x\|^{p}}: x \in E,\|x\| \geq 1\right\}
$$

is unbounded, then $f$ satisfies af $(x+y)=f(x) f(y)$ for all $x, y \in E$. Moreover, if $a=0$, then $f \equiv 0$.

Proof We assume that $\varepsilon+\theta>0$ and $O_{R E}(f, b)$ is unbounded for each $b \neq 0$. We continue to employ the notation $\sharp a \sharp$, to denote $\|a\|$ (if $a \in \mathcal{A}$ ) and $|a|$ (if $a \in \mathbb{R}$ ), respectively. For every $x, y, z \in E$, we have

$$
\begin{aligned}
\|f(x)[f(y) f(z)-a f(y+z)]\| \leq & \left\|a^{2} f(x+y+z)-a f(x) f(y+z)\right\| \\
& +\left\|a f(x+y) f(z)-a^{2} f(x+y+z)\right\| \\
& +\|f(x) f(y) f(z)-a f(x+y) f(z)\| \\
\leq & \varepsilon \sharp a \sharp\left(\|x\|^{p}+\|y+z\|^{p}+\|x+y\|^{p}+\|z\|^{p}\right) \\
& +\theta \sharp a \sharp\left(\|x\|^{p}\|y+z\|^{p}+\|x+y\|^{p}\|z\|^{p}\right) \\
& +\varepsilon\|f(z)\|\left(\|x\|^{p}+\|y\|^{p}\right)+\theta\|f(z)\|\|x\|^{p}\|y\|^{p} \\
\leq & \left(\|f(z)\|+2^{p+1} \sharp a \sharp\right) \varepsilon\left(\|x\|^{p}+\|y\|^{p}+\|z\|^{p}\right) \\
& +\left(\|f(z)\|+2^{p} \sharp a \sharp+2^{p}\right) \\
& \times \theta\left(\|x\|^{p}\|y\|^{p}+\|x\|^{p}\|z\|^{p}+\|y\|^{p}\|z\|^{p}\right) .
\end{aligned}
$$

Then, for $\|x\| \geq 1$, we have

$$
\begin{aligned}
\|f(x)[f(y) f(z)-a f(y+z)]\| \leq & \left(\|f(z)\|+2^{p+1} \sharp a \sharp\right) \varepsilon\|x\|^{p}\left(1+\|y\|^{p}+\|z\|^{p}\right) \\
& +\left(\|f(z)\|+2^{p} \sharp a \sharp+2^{p}\right) \theta\|x\|^{p}\left(\|y\|^{p}+\|z\|^{p}+\|y\|^{p}\|z\|^{p}\right) \\
= & M\|x\|^{p},
\end{aligned}
$$

where

$$
\begin{aligned}
M:= & \varepsilon\left(\|f(z)\|+2^{p+1} \sharp a \sharp\right)\left(1+\|y\|^{p}+\|z\|^{p}\right) \\
& +\theta\left(\|f(z)\|+2^{p} \sharp a \sharp+2^{p}\right)\left(\|y\|^{p}+\|z\|^{p}+\|y\|^{p}\|z\|^{p}\right) .
\end{aligned}
$$

Therefore,

$$
\left\|f(x) \frac{f(y) f(z)-a f(y+z)}{M}\right\| \leq\|x\|^{p} .
$$

Letting $b:=\frac{f(y) f(z)-a f(y+z)}{M}$, we get $O_{R E}(f, b)$ is bounded. By assumption, this implies $b=0$. Hence $a f(y+z)=f(y) f(z)$. Moreover, if $a=0$, then we get $f(x) f(y)=0$ for all $x, y \in E$. Let $y \in E$ be an arbitrary element. Then $O_{R E}(f, f(y))=\{0\}$ is bounded, and by assumption we conclude that $f(y)=0$. Hence $f \equiv 0$. If we assume that $O_{L E}(b)$ is unbounded for each nonzero $b \in \mathcal{A}$, the proof proceeds in a similar way. 
Corollary 3.6 Let $E$ be a normed linear space and $\mathcal{A}$ be a commutative semisimple complex Banach algebra. Assume that a mapping $f: E \rightarrow$ A satisfies

$$
\|f(x+y)-f(x) f(y)\| \leq \varepsilon\left(\|x\|^{p}+\|y\|^{p}\right)+\theta\|x\|^{p}\|y\|^{p}, \quad x, y \in E
$$

for some $\varepsilon, \theta, p \geq 0$. If, for every nonzero linear multiplicative functional $\varphi$ on $\mathcal{A}$, the set

$$
G_{\varphi}:=\left\{\frac{(\varphi \circ f)(x)}{\|x\|^{p}}: x \in E,\|x\| \geq 1\right\}
$$

is unbounded, then $f$ is exponential.

Proof Let $b \neq 0$ be an element in $\mathcal{A}$. Since $\mathcal{A}$ is semisimple, there is a linear multiplicative functional $\varphi$ on $\mathcal{A}$ such that $\varphi(b) \neq 0$. By assumption, $G_{\varphi}$ is unbounded. Then the set

$$
G_{\varphi} \cdot \varphi(b)=\left\{\frac{\varphi(f(x) b)}{\|x\|^{p}}: x \in E,\|x\| \geq 1\right\}=\varphi\left(O_{R E}(b)\right)
$$

is unbounded, and we conclude that $O_{R E}(b)$ is unbounded. By Theorem 3.5, $f$ is exponential.

Corollary 3.7 Let E be a normed linear space and $\mathcal{A}$ be a complex Banach algebra. Assume that mappings $f, g: E \rightarrow \mathcal{A}$ satisfy $f(0) f(z)=f(z) f(0)$ for all $z \in E$, and (3.7). If, for each nonzero element $b \in \mathcal{A}$, the E-orbit of $b$

$$
O_{R E}(f, b):=\left\{\frac{f(x) b}{\|x\|^{p}}: x \in E,\|x\| \geq 1\right\} \quad \text { or } \quad O_{L E}(b, f):=\left\{\frac{b f(x)}{\|x\|^{p}}: x \in E,\|x\| \geq 1\right\}
$$

is unbounded, then $f$ satisfies $f(0) f(x+y)=f(x) f(y)$ for all $x, y \in E$. Moreover, if $f(0)=0$, then $f \equiv 0$.

Proof As in the proof of Theorem 3.2, we obtain

$$
\begin{aligned}
\|f(0) f(x+y)-f(x) f(y)\| & \leq\|f(0) f(x+y)-g(x+y)\|+\|g(x+y)-f(x) f(y)\| \\
& \leq\left(2^{p}+1\right) \varepsilon\left(\|x\|^{p}+\|y\|^{p}\right)+\theta\|x\|^{p}\|y\|^{p}, \quad x, y \in E .
\end{aligned}
$$

By Theorem 3.5, we get the desired result.

Theorem 3.8 Let E be a normed linear space and $\mathcal{A}$ be a complex Banach algebra. Assume that $\varepsilon, \theta, p \geq 0, a \in \mathcal{A} \cup \mathbb{R}$ and $f, g: E \rightarrow \mathcal{A}$ satisfy one of the following conditions:

(i) $a f(z)=f(z) a,\|a f(x+y)-f(x) g(y)\| \leq \varepsilon\left(\|x\|^{p}+\|y\|^{p}\right)+\theta\|x\|^{p}\|y\|^{p}, x, y, z \in E$; and for each nonzero element $b \in \mathcal{A}$, the $E$-orbit of $b$

$$
O_{R E}(f, b):=\left\{\frac{f(x) b}{\|x\|^{p}}: x \in E,\|x\| \geq 1\right\}
$$

is unbounded. 
(ii) $a g(z)=g(z) a,\|a f(x+y)-g(x) f(y)\| \leq \varepsilon\left(\|x\|^{p}+\|y\|^{p}\right)+\theta\|x\|^{p}\|y\|^{p}, x, y, z \in E$; and for each nonzero element $b \in \mathcal{A}$, the E-orbit of $b$

$$
O_{L E}(b, f):=\left\{\frac{b f(x)}{\|x\|^{p}}: x \in E,\|x\| \geq 1\right\}
$$

is unbounded.

Then $g$ satisfies $a g(x+y)=g(x) g(y)$ for all $x, y \in E$. Moreover, if $a=0$, then $g \equiv 0$.

Proof Let $f, g$ satisfy (i) and $O_{R E}(f, b)$ be unbounded for each nonzero element $b \in \mathcal{A}$. Using the same argument as in the proof of Theorem 3.3, we obtain

$$
\begin{aligned}
\|a f(x) g(y+z)-f(x) g(y) g(z)\| \leq & \varepsilon \sharp a \sharp\left(\|x+y\|^{p}+\|y+z\|^{p}+\|x\|^{p}+\|z\|^{p}\right) \\
& +\theta \sharp a \sharp\left(\|x+y\|^{p}\|z\|^{p}+\|x\|^{p}\|y+z\|^{p}\right) \\
& +\|g(z)\|\left[\varepsilon\left(\|x\|^{p}+\|y\|^{p}\right)+\theta\|x\|^{p}\|y\|^{p}\right]
\end{aligned}
$$

for all $x, y, z \in E$. Since $a f(x)=f(x) a$, for $\|x\| \geq 1$, we obtain

$$
\begin{aligned}
\|f(x)[a g(y+z)-g(y) g(z)]\| \leq & \left(\|g(z)\|+2^{p+1} \sharp a \sharp\right) \varepsilon\|x\|^{p}\left(1+\|y\|^{p}+\|z\|^{p}\right) \\
& +\left(\|g(z)\|+2^{p} \sharp a \sharp+2^{p}\right) \theta\|x\|^{p}\left(\|y\|^{p}+\|z\|^{p}+\|y\|^{p}\|z\|^{p}\right) \\
= & M\|x\|^{p}
\end{aligned}
$$

where

$$
\begin{aligned}
M:= & \varepsilon\left(\|g(z)\|+2^{p+1} \sharp a \sharp\right)\left(1+\|y\|^{p}+\|z\|^{p}\right) \\
& +\theta\left(\|g(z)\|+2^{p} \sharp a \sharp+2^{p}\right)\left(\|y\|^{p}+\|z\|^{p}+\|y\|^{p}\|z\|^{p}\right) .
\end{aligned}
$$

Therefore

$$
\left\|f(x) \frac{a g(y+z)-g(y) g(z)}{M}\right\| \leq\|x\|^{p} .
$$

Letting $b:=\frac{a g(y+z)-g(y) g(z)}{M}$, by assumption, we get that $b=0$. Therefore $a g(y+z)=g(y) g(z)$ for all $y, z \in E$. Moreover, if $a=0$, then (i) implies that $g \equiv 0$.

Similarly, we get the result if $f, g$ satisfy condition (ii).

Theorem 3.9 Let $E$ be a normed linear space and $\mathcal{A}$ be a complex Banach algebra. Let $f, g, h: E \rightarrow \mathcal{A}$ satisfy the inequality

$$
\|f(x+y)-g(x) h(y)\| \leq \varepsilon\left(\|x\|^{p}+\|y\|^{p}\right)+\theta\|x\|^{p}\|y\|^{p}, \quad x, y \in E
$$

for some $\varepsilon, \theta, p \geq 0$.

(i) If $g(0) g(x)=g(x) g(0)$ for all $x \in E$, and for each nonzero element $b \in \mathcal{A}$ the E-orbit of $b$

$$
O_{L E}(b, h):=\left\{\frac{b h(x)}{\|x\|^{p}}: x \in E,\|x\| \geq 1\right\}
$$


is unbounded, then

$$
g(0) g(x+y)=g(x) g(y), \quad x, y \in E .
$$

Moreover, if $g(0)=0$, then $g \equiv 0$.

(ii) If $h(0) g(x)=g(x) h(0)$ for all $x \in E$, and for each nonzero element $b \in \mathcal{A}$ the E-orbit of $b$

$$
O_{R E}(g, b):=\left\{\frac{g(x) b}{\|x\|^{p}}: x \in E,\|x\| \geq 1\right\}
$$

is unbounded, then

$$
h(0) h(x+y)=h(x) h(y), \quad x, y \in E .
$$

Moreover, if $h(0)=0$, then $h \equiv 0$.

Proof Using the same argument as in the proof of Theorem 3.4, we obtain

$$
\|g(0) h(x+y)-g(x) h(y)\| \leq\left(2^{p}+1\right) \varepsilon\left(\|x\|^{p}+\|y\|^{p}\right)+\theta\|x\|^{p}\|y\|^{p}
$$

and

$$
\|g(x+y) h(0)-g(x) h(y)\| \leq\left(2^{p}+1\right) \varepsilon\left(\|x\|^{p}+\|y\|^{p}\right)+\theta\|x\|^{p}\|y\|^{p}
$$

for all $x, y, z \in E$. Therefore the result follows from Theorem 3.8.

Remark 3.10 We can replace $\varepsilon\left(\|x\|^{p}+\|y\|^{p}\right)+\theta\|x\|^{p}\|y\|^{p}$ given in the main results of this section by more general control functions $\varphi(x, y)$ given by Găvruta [22]. The proofs are similar to the proofs given in this section.

\section{Conclusion}

We have proved the superstability of the following functional equations:

$$
\begin{aligned}
& f(P(x, y))=g(x) h(y), \\
& f(x+y)=g(x) h(y) .
\end{aligned}
$$

Acknowledgements

We would like to express our sincere gratitude to the anonymous referee for his/her helpful comments that will help to improve the quality of the manuscript.

Funding

Not applicable

\section{Availability of data and materials}

Not applicable.

Ethics approval and consent to participate

We would like to mention that this article does not contain any studies with animals and does not involve any studies over human being. 
Competing interests

The authors declare that they have no competing interests.

\section{Authors' contributions}

The authors equally conceived of the study, participated in its design and coordination, drafted the manuscript, participated in the sequence alignment, and read and approved the final manuscript.

\section{Publisher's Note}

Springer Nature remains neutral with regard to jurisdictional claims in published maps and institutional affiliations.

Received: 24 December 2020 Accepted: 15 April 2021 Published online: 23 April 2021

\section{References}

1. Ulam, S.M.: Problems in Modern Mathematics. Wiley, New York (1964)

2. Hyers, D.H.: On the stability of the linear functional equation. Proc. Natl. Acad. Sci. USA 27, 222-224 (1941)

3. Ding, Y., Xu, T.Z.: Approximate solution of generalized inhomogeneous radical quadratic functional equations in 2-Banach spaces. J. Inequal. Appl. 2019, 31 (2019)

4. Park, C., Bodaghi, A.: Two multi-cubic functional equations and some results on the stability in modular spaces. J. Inequal. Appl. 2020, 6 (2020)

5. Jung, S., Popa, D., Rassias, M.T.: On the stability of the linear functional equation in a single variable on complete metric spaces. J. Glob. Optim. 59, 13-16 (2014)

6. Lee, Y., Jung, S., Rassias, M.T.: Uniqueness theorems on functional inequalities concerning cubic-quadratic-additive equation. J. Math. Inequal. 12,43-61 (2018)

7. Shtern, A.I.: Exponential stability of quasihomomorphisms into Banach algebras and a Ger-Šemrl theorem. Russ. J. Math. Phys. 22, 141-142(2015)

8. Madadi, M., Saadati, R., Rassias, J.M.: Stochastic Lie bracket (derivation, derivation) in MB-algebras. J. Inequal. Appl. 2020, $141(2020)$

9. Naeem, R., Anwar, M.: Weighted Jessen's functionals and exponential convexity. J. Math. Comput. Sci. 19, 171-180 (2019)

10. Abolghasemi, M., Khoei, M.A.: Bounded and sequential $\sigma$-approximate amenability of Banach algebras. J. Math. Comput. Sci. 18, 248-254 (2018)

11. Baker, J., Lawrence, J., Zorzitto, F.: The stability of the equation $f(x+y)=f(x) f(y)$. Proc. Am. Math. Soc. 74, 242-246 (1979)

12. Baker, J.: The stability of the cosine equation. Proc. Am. Math. Soc. 80, 411-416 (1980)

13. Găvruta, P.: On the stability of some functional equation. In: Stability of Mappings of Hyers-Ulam Type, pp. 93-98. Hardronic Press, Palm Harbor (1994)

14. Kim, G.: Stability of the Pexiderized Lobacevski equation. J. Appl. Math. 2011, Article ID 540274 (2011)

15. Kim, G., Park, C.: Superstability of an exponential equation in $C^{*}$-algebras. Results Math. 67, 197-205 (2015)

16. Keltouma, B., Elhoucien, E., Rassias, T.M., Ahmed, R.: Superstability of Kannappan's and Van Vleck's functional equations. J. Nonlinear Sci. Appl. 11, 894-915 (2018)

17. Kim, G., Lee, Y.: Superstability of Pexiderized functional equations arising from distance measures. J. Nonlinear Sci. Appl. 9, 413-423 (2016)

18. Saadati, R., Park, C.: Approximation of derivations and the superstability in random Banach $*$-algebras. Adv. Differ. Equ. $2018,418(2018)$

19. Dixmier, J.: ( ${ }^{*}$-algebras. North-Holland, Amsterdam (1977)

20. Székelyhidi, L.: On a theorem of Baker, Lawrence and Zorzitto. Proc. Am. Math. Soc. 84, 95-96 (1982)

21. Găvruta, P.: An answer to a question of Th. M. Rassias and J. Tabor on mixed stability of mappings. Bul. Ştiinţ. Univ. "Politehnica" Timiş., Ser. Mat.-Fiz. 42, 1-6 (1997)

22. Găvruta, P.: A generalization of the Hyers-Ulam-Rassias stability of approximately additive mappings. J. Math. Anal. Appl. 184, 431-436 (1994)

\section{Submit your manuscript to a SpringerOpen ${ }^{\circ}$ journal and benefit from:}

- Convenient online submission

- Rigorous peer review

- Open access: articles freely available online

- High visibility within the field

Retaining the copyright to your article

Submit your next manuscript at $>$ springeropen.com 